\title{
On Optimal Input Signal Design for Identification of Output Error Models
}

\author{
Bo Wahlberg, Mariette Annergren and Cristian R. Rojas
}

\begin{abstract}
This paper extends recent results on minimum variance input signal design for identification of Finite Impulse Response (FIR) models to the Output Error (OE) system identification case. The idea is to use "the useful input parametrization" for OE models proposed by Stoica and Söderstrom (1982). The advantage of this parametrization is that the Toeplitz covariance matrix structure instrumental in the FIR analysis also holds for this $O E$ model input representation after a transformation. However, an issue is that the corresponding minimum variance cost function for the $\mathrm{OE}$ case will be more complicated than for FIR models, and that the dimension of the optimization problem will be of one degree higher than for the corresponding FIR case. The proposed OE framework is applied to minimum variance input signal design in system identification frequency response estimation and model predictive control. The results are illustrated by numerical examples.
\end{abstract}

\section{INTRODUCTION}

In recent years there have been considerable advances in experiment design for identification of dynamical systems based on convex optimization methods, [8], [6], [16], [3], [15], [21], [7], [19], [1], [4], [9], [14], [10], [2], [5], [13], [12]. As shown in [20] many classical methods for input design can be reformulated as convex optimization problems. The unifying framework for optimal input design for system identification presented in [11] provides a transparent way to connect the performance of the estimated model in the intended application to the system identification experiment conditions. The identification objective is to guarantee, with a given probability, that the estimated model will be in the set of models that satisfies given specifications. The papers [23], [24] have analyzed and evaluated this framework, in detail, by using the fact that the corresponding input minimum variance optimization problem has a very simple structure for Finite Impulse Response (FIR) models. The problem is more involved for Output Error (OE), even if it is possible to find the optimal input for such models using numerical convex optimization. However, by using the input parametrization proposed in [22], several structural FIR results can be extended to identification of $\mathrm{OE}$, and also BoxJenkins, models. This parametrization also allows for a direct implementation of the corresponding SDP/LMI optimization problem.

The outline of the paper is as follows: In Section II application motivated system identification and optimal input design are introduced. Section III considers the output error model case in detail. Frequency response estimation is studied in Section IV, while Section V and VI deals with control application examples. Section VII concludes the paper.

This work was partially supported by the Swedish Research Council and the Linnaeus Center ACCESS at KTH. The research leading to these results has received funding from The European Research Council under the European Community's Seventh Framework program (FP7 2007-2013) / ERC Grant Agrement N. 267381

Automatic Control Lab and ACCESS, School of Electrical Engineering, KTH Royal Institute of Technology, SE-100 44 Stockholm, Sweden. (e-mail: bo.wahlberg@ee.kth.se, mariette.annergren@ee.kth.se, cristian.rojas@ee.kth.se.)

\section{PROBlem Formulation}

Consider an asymptotically stable time-invariant discretetime linear dynamical system

$$
y(t)=\frac{B(q)}{F(q)} u(t)+e(t),
$$

with input signal sequence $\{u(t)\}$, output signal sequence $\{y(t)\}$, and zero mean white noise $\{e(t)\}$ with variance $\sigma^{2}$. Here

$B(q)=b_{1} q^{-1}+\ldots+b_{n} q^{-n}, F(q)=1+f_{1} q^{-1}+\ldots+f_{n} q^{-n}$,

where $q^{-1}$ is the delay operator, i.e. $q^{-1} u(t)=u(t-1)$. It is assumed that the roots of $F(q)$ are strictly inside the unit circle and that $B(q)$ and $F(q)$ have no common factor (no pole zero cancelations). The relation (1) is called an Output Error (OE) model structure. By also including a noise model with a separate parametrization we can extend the results to be presented to so-called Box-Jenkins models.

We are interested in applications that depend on the transfer function $G(q)=B(q) / F(q)$. One important example is the frequency response $G\left(e^{i \omega}\right)=B\left(e^{i \omega}\right) / F\left(e^{i \omega}\right)$. Other applications are model based control design, such as internal model control and model predictive control.

Introduce the model parameter vector

$$
\boldsymbol{\theta}=\left[b_{1}, \ldots, b_{n}, f_{1}, \ldots, f_{n}\right]^{T} \in \mathbb{R}^{2 n},
$$

and assume that the true system can be described by the parameter vector $\theta_{o}$. We will use the notation $G(q, \theta)=$ $B(q, \theta) / F(q, \theta)$ to stress the parameter dependence.

\section{A. Application}

Let $V_{a p p}(\theta)$ denote an application cost function that measures the degradation in performance due to model errors for a certain model based application. The specifications are given by

$$
V_{\text {app }}(\theta) \leq \frac{1}{2 \gamma}
$$

for some constant $\gamma>0$. Without loss of generality, we can assume that $V_{a p p}(\theta) \geq 0$ with minimum at $\theta_{o}$ such that $V_{a p p}\left(\theta_{o}\right)=0$. This leads to the Taylor series expansion approximation

$$
V_{a p p}(\theta) \approx 0.5\left[\theta-\theta_{o}\right]^{T} V_{a p p}^{\prime \prime}\left(\theta_{o}\right)\left[\theta-\theta_{o}\right],
$$

where we have neglected higher order terms. The application specifications (2) can thus be approximated by the ellipsoidal set

$$
\mathscr{E}_{a p p}=\left\{\theta:\left[\theta-\theta_{o}\right]^{T} V_{a p p}^{\prime \prime}\left(\theta_{o}\right)\left[\theta-\theta_{o}\right] \leq \frac{1}{\gamma}\right\} .
$$

The accuracy of this approximation depends on $V_{a p p}(\theta)$ and $\gamma$. A high value of $\gamma$ gives a more restricted set and a better quadratic approximation. 
For the frequency response example we have

$$
V_{\text {app }}(\theta)=\left|G\left(e^{i \omega}, \theta\right)-G\left(e^{i \omega}, \theta_{o}\right)\right|^{2} .
$$

\section{B. System Identification}

Let $\hat{\theta}_{N}$ be a model parameter vector estimated from $N$ measured input output observations, $\{u(t), y(t), t=1, \ldots, N\}$ using a Prediction Error Model (PEM) identification method, see [17]. We then have the following asymptotic (large $N$ ) result

$$
\hat{\theta}_{N} \in \mathscr{E}_{S I}=\left\{\theta:\left[\theta-\theta_{o}\right]^{T} \mathbf{I}_{F}\left[\theta-\theta_{o}\right] \leq \frac{\kappa}{N}\right\}, \quad \text { w.p. } \alpha,
$$

where

$$
\mathbf{I}_{F}=\frac{1}{2 \pi} \int_{-\pi}^{\pi} \Psi_{u}\left(\mathrm{e}^{i \omega}\right) \Psi_{u}^{*}\left(\mathrm{e}^{i \omega}\right) \mathrm{d} \omega,
$$

is the average Fisher information matrix and $\Psi_{u}\left(\mathrm{e}^{i \omega}\right)$ is the normalized prediction error gradient. The constant $\kappa$ depends on the number of estimated parameters and the probability $\alpha$, and can be determined from the $\chi^{2}$-distribution. This means that we guarantee with probability $\alpha$ that the estimated model belongs to the ellipsoidal set given in (4). Furthermore, (provided the input is persistently exciting so that the Fisher information matrix is invertible)

$$
\mathrm{E}\left\{\left[\hat{\theta}_{N}-\theta_{o}\right]\left[\hat{\theta}_{N}-\theta_{o}\right]^{T}\right\} \approx \frac{1}{N} \mathbf{I}_{F}^{-1},
$$

for large $N$.

In Section III we will in detail discuss the Fisher information matrix $\mathbf{I}_{F}$ for OE models.

\section{Minimum Variance Input Design}

To connect system identification to the application we need to insure that the system identification set defined by (4) is inside the application set defined by the quadratic form (3). Since both sets are ellipsoidal, it is enough to verify the matrix inequality ${ }^{1}$

$$
\frac{N}{\kappa} \mathbf{I}_{F} \geq \gamma V_{a p p}^{\prime \prime}\left(\theta_{o}\right) .
$$

This inequality together with (4) imply $\hat{\theta}_{N} \in \mathscr{E}_{\text {app }}$, (3), with at least probability $\alpha$.

Inspired by the framework of least costly identification experiment design for control introduced in [5], the following minimum variance input signal optimization problem

$$
\min _{u(t)} \mathrm{E}\left\{u^{2}(t)\right\} \quad \text { s.t. } \quad \frac{N}{\kappa} \mathbf{I}_{F} \geq \gamma V_{a p p}^{\prime \prime}\left(\theta_{o}\right),
$$

where $\{u(t)\}$ is the input signal to be used in the system identification experiment, where proposed in [11]. The objective is to find the minimum variance input signal such that the application specification is satisfied for the estimated model $\hat{\theta}_{N}$. It is important to understand how the Fisher information matrix $\mathbf{I}_{F}$ depends on the input signal. In general, $\mathbf{I}_{F}$ is an affine function in the input power spectral density $\Phi_{u}(\omega)$ and since $\mathrm{E}\left\{u^{2}(t)\right\}$ is also linear in $\Phi_{u}(\omega)$, we obtain a Semi-Definite Program (SDP) (linear cost-function and linear matrix inequities) where we optimize with respect to the power spectral density or the corresponding covariance function. This infinite dimensional convex optimization problem can be approximately solved using a finite dimensional parametrization of $\Phi(\omega) \geq 0$. The problem simplifies greatly

\footnotetext{
${ }^{1} X \geq Y$ means that $[X-Y]$ is a positive semi-definite matrix.
}

for Finite Impulse Response (FIR) models, since $\sigma^{2} \mathbf{I}_{F}$ is the symmetric Toeplitz matrix with the initial values of the covariance function of $\{u(t)\}, r_{k}=\mathrm{E}\{u(t) u(t-k)\}$, as its first row. Since the cost function is the variance of $\{u(t)\}$, i.e. $r_{0}$, we obtain a Semi-Definite Program with optimization variables $r_{k}, k=0, \ldots,(n-1)$.

A more classical formulation of the minimum variance input signal design problem is

$$
\min _{u(t)} \mathrm{E}\left\{u^{2}(t)\right\} \quad \text { s.t. } \quad \mathrm{E}\left\{V_{a p p}\left(\hat{\theta}_{N}\right)\right\} \leq \gamma .
$$

This problem can be approximated by

$$
\min _{u(t)} \mathrm{E}\left\{u^{2}(t)\right\} \quad \text { s.t. } \quad \operatorname{trace}\left\{V_{a p p}^{\prime \prime}\left(\theta_{o}\right) \frac{1}{N} \mathbf{I}_{F}^{-1}\right\} \leq \gamma,
$$

and can also be reformulated as convex optimization problem using Schur complements.

\section{Structural Results fOR OE Models}

For OE models the gradient filter (without normalization)

$$
\Psi(q)=\frac{d}{d \theta} G(q, \theta)
$$

has the following structure, see [17],

$$
\begin{aligned}
& \Psi(q)= \\
& {\left[\frac{q^{-1}}{F(q)}, \ldots, \frac{q^{-n}}{F(q)}, \frac{B(q)}{F(q)} \frac{\left(-q^{-1}\right)}{F(q)}, \ldots, \frac{B(q)}{F(q)} \frac{\left(-q^{-n}\right)}{F(q)}\right]^{T},}
\end{aligned}
$$

and the Information Matrix equals

$$
\mathbf{I}_{F}=\frac{1}{\sigma^{2}} \mathrm{E}\left\{[\Psi(q) u(t)][\Psi(q) u(t)]^{T}\right\} .
$$

The key idea, introduced in [22], is to write

$$
\begin{aligned}
& \Psi(q)=\frac{1}{F^{2}(q)} \times \\
& {\left[q^{-1} F(q), \ldots, q^{-n} F(q),\left(-q^{-1}\right) B(q), \ldots,\left(-q^{-n}\right) B(q)\right]^{T}} \\
& =\mathscr{S}(F,-B)\left[q^{-1}, \ldots, q^{-2 n}\right]^{T} \frac{1}{F^{2}(q)}
\end{aligned}
$$

where we have introduced the Sylvester matrix

$$
\mathscr{S}(F,-B)=\left[\begin{array}{ccccccc}
1 & f_{1} & & \ldots & f_{n} & & \\
0 & \ddots & \ddots & & & \ddots & 0 \\
0 & -b_{1} & & f_{1} & \ldots & & f_{n} \\
0 & \ddots & \ddots & & & \ddots & 0 \\
& & 0 & -b_{1} & \ldots & & -b_{n}
\end{array}\right] .
$$

Define the filtered input signal

$$
\bar{u}(t)=\frac{1}{F^{2}(q)} u(t)
$$

for which

$$
\Psi(q) u(t)=\mathscr{S}(F,-B)[\bar{u}(t-1) \bar{u}(t-2), \ldots, \bar{u}(t-2 n)]^{T}
$$

and thus

$$
\mathbf{I}_{F}=\frac{1}{\sigma^{2}} \mathscr{S}\left(F_{o},-B_{o}\right) \bar{R}_{2 n} \mathscr{S}\left(F_{o},-B_{o}\right)^{T} .
$$

The matrix $\bar{R}_{2 n}$ is the $2 n \times 2 n$ symmetric Toeplitz matrix with first row $\left[\bar{r}_{0}, \ldots \bar{r}_{2 n-1}\right]$, where $\bar{r}_{k}=\mathrm{E}\{\bar{u}(t) \bar{u}(t-k)\}$ is 
the covariance function of the filtered input signal $\bar{u}(t)$. We have. as before, denoted the true system with sub-index $o$.

In the frequency domain

$$
\Psi_{u}\left(\mathrm{e}^{i \omega}\right)=\Psi\left(\mathrm{e}^{i \omega}\right) \frac{\sqrt{\Phi_{u}\left(\mathrm{e}^{i \omega}\right)}}{\sigma}
$$

in (5), where $\Phi_{u}\left(\mathrm{e}^{i \omega}\right)$ is the power spectral density of $\{u(t)\}$.

In order to formulate the minimum variance optimal input signal design problem we have to relate the variance of the original input signal $u(t)$ to the covariance function of $\bar{u}(t)$,

$$
\mathrm{E}\left\{u^{2}(t)\right\}=\mathrm{E}\left\{\left[F^{2}\left(q, \theta_{o}\right) \bar{u}(t)\right]^{2}\right\}=\bar{f}_{o}^{T} \bar{R}_{2 n+1} \bar{f}_{o}
$$

where $\bar{f}_{o}$ is the $n+1$ dimensional column vector constructed from the coefficients of $F^{2}\left(q, \theta_{o}\right)$. Notice that we have to increase the dimension of the Toeplitz matrix to $(n+1) \times$ $(n+1)$ to determine the variance of $u(t)$. This means that we need to optimize with respect to the covariances $\bar{r}_{0}$ to $\bar{r}_{2 n}$ of $\bar{u}(t)$.

Another option is to minimize the variance of the output signal $y(t)$, c.f. [18], by using

$$
\mathrm{E}\left\{y^{2}(t)\right\}=\mathrm{E}\left\{\left[B\left(q, \theta_{o}\right) F\left(q, \theta_{o}\right) \bar{u}(t)\right]^{2}\right\}=\bar{b}_{o}^{T} \bar{R}_{2 n} \bar{b}_{o},
$$

where $\bar{b}_{o}$ is the $n$ dimensional column vector constructed from the coefficients of the polynomial $B\left(q, \theta_{o}\right) F\left(q, \theta_{o}\right)$. Notice that the leading coefficient of $B(q) F(q)$ is zero and we thus only need to know the covariances $\bar{r}_{k}, k=0, \ldots,(2 n-1)$ to calculate the variance of $y(t)$, i.e. the same number of covariances that is defining $\mathbf{I}_{F}$.

\section{A. Optimal Input Design for OE Models}

We can now write the constraint in the minimum variance input optimization problem (9) for OE models as

$$
\frac{N}{\kappa \sigma^{2}} \mathscr{S}\left(F_{o},-B_{o}\right) \bar{R}_{2 n} \mathscr{S}\left(F_{o},-B_{o}\right)^{T} \geq \gamma V_{a p p}^{\prime \prime}\left(\theta_{o}\right) .
$$

Since we assume no pole zero cancelations for the true system, the Sylvester matrix $\mathscr{S}\left(F_{o},-B_{o}\right)$ has full rank. Hence, we can write (9) as

$$
\begin{array}{ll} 
& \min \bar{f}_{o}^{T} \bar{R}_{2 n+1} \bar{f}_{o} \\
\text { s.t. } & \bar{R}_{2 n} \geq \frac{\kappa \sigma^{2} \gamma}{N} \mathscr{S}\left(F_{o},-B_{o}\right)^{-1} V_{a p p}^{\prime \prime}\left(\theta_{o}\right) \mathscr{S}\left(F_{o},-B_{o}\right)^{-T} \\
& \bar{R}_{2 n+1} \geq 0 .
\end{array}
$$

We have to add the constraint $\bar{R}_{2 n+1} \geq 0$ for $\bar{r}_{k}, k=0, \ldots, 2 n$, to be a quasi-stationary covariance sequence.

If we instead choose to minimize the variance of the output signal $y(t)$, we should solve

$$
\begin{array}{ll} 
& \min \bar{r}_{k}, k=0, \ldots, 2 n-1 \\
\text { s.t. } & \bar{R}_{2 n}^{T} \geq \frac{\kappa \sigma^{2} \gamma}{N} \mathscr{S}\left(F_{o},-B_{o}\right)^{-1} V_{a p p}^{\prime \prime}\left(\theta_{o}\right) \mathscr{S}\left(F_{o},-B_{o}\right)^{-T} .
\end{array}
$$

It is easy to recover $u(t)$ from $\bar{u}(t)$ by using $u(t)=F^{2}(q) u(t)$.

These two optimization problems look very much as the one for optimal input design for identification of FIR models, and both can be solved by more or less re-using the FIR code. The two main differences are
- The cost function $\bar{f}^{T} \bar{R}_{2 n+1} \bar{f}^{T}$ is slightly more complicated than for the FIR case, where we just minimize the input variance $r_{0}$.

- For the OE case the dimension of the optimization problem for input variance minimization equals the number of parameters plus one. It is one dimension higher than for the corresponding FIR problem. This implies that it is difficult to obtain an analytic solution even for the simplest OE model case with just two parameters.

\section{FREQUENCy RESPONSE ESTIMATION}

We will start by analyzing the classical frequency response problem for which

$$
V_{\text {app }}(\theta)=\left|G\left(e^{i \omega}, \theta\right)-G\left(e^{i \omega}, \theta_{o}\right)\right|^{2},
$$

where $\omega$ is a given frequency. For

$$
\begin{aligned}
G\left(e^{i \omega}, \theta\right) & =\frac{B\left(e^{i \omega}, \theta\right)}{F\left(e^{i \omega}, \theta\right)} \Rightarrow \\
\frac{d}{d \theta} G\left(e^{i \omega}, \theta\right) & =\mathscr{S}(F,-B)\left[e^{-i \omega} \ldots e^{-2 n i \omega}\right]^{T} \frac{1}{F^{2}\left(e^{i \omega}, \theta\right)} .
\end{aligned}
$$

Here we have used the same calculations as when deriving the gradient filter $\Psi(q)$ in (10). Now

$$
\begin{aligned}
& V_{\text {app }}^{\prime \prime}\left(\theta_{o}\right)=\frac{2}{\left|F\left(e^{i \omega}, \theta_{o}\right)\right|^{4}} \mathscr{S}\left(F_{o},-B_{o}\right) \times \\
& \quad \operatorname{Re}\left\{\left[e^{-i \omega}, \ldots, e^{-2 n i \omega}\right]^{*}\left[e^{-i \omega}, \ldots, e^{-2 n i \omega}\right]\right\} \mathscr{S}\left(F_{o},-B_{o}\right)^{T} .
\end{aligned}
$$

Here $\operatorname{Re}$ denotes the real part of the matrix. Notice that $V_{a p p}\left(\theta_{o}\right)$ is a real-valued function of $\theta \in \mathbb{R}^{2 n}$. We now obtain the simple expression

$$
\mathscr{S}\left(F_{o},-B_{o}\right)^{-1} V_{a p p}^{\prime \prime}\left(\theta_{o}\right) \mathscr{S}\left(F_{o},-B_{o}\right)^{-T}=\frac{2}{\left|F\left(e^{i \omega}, \theta_{o}\right)\right|^{4}} M,
$$

where $M$ is the symmetric $2 n \times 2 n$ Toeplitz matrix with first row $[1, \cos (\omega), \ldots, \cos ((2 n-1) \omega)]$.

We now have the input minimum variance problem $\left(\min \mathrm{E}\left\{u^{2}(t)\right\}\right)$

$$
\begin{array}{ll} 
& \min \bar{r}_{k}, k=0, \ldots, 2 n \\
\text { s.t. } & \bar{R}_{2 n}^{T} \geq \frac{\kappa \sigma^{2} \gamma}{N} \frac{2}{\left|F\left(e^{i \omega}, \theta_{o}\right)\right|^{4}} M, \quad \bar{R}_{2 n+1} \geq 0
\end{array}
$$

and the output minimum variance problem $\left(\min \mathrm{E}\left\{y^{2}(t)\right\}\right)$

$$
\begin{array}{ll} 
& \min \bar{r}_{k}, k=0, \ldots, 2 n-1 \\
\text { s.t. } & \bar{R}_{2 n} \geq \frac{\kappa \sigma^{2} \gamma}{N} \frac{2}{\left|F\left(e^{i \omega}, \theta_{o}\right)\right|^{4}} M .
\end{array}
$$

For $\omega \neq 0, \pi$ the Toeplitz matrix $M$ is the covariance matrix corresponding to the sinusoidal signal

$$
m(t)=\sqrt{2} \cos (\omega t) \quad \Rightarrow \quad r_{k}=\cos (\omega k), k=0,1, \ldots
$$

Here we have used the framework of quasi-stationary signals to define the covariance function $r_{k}$ of the sinusoidal signal $m(t)$, see [17]. (16).

It is easy to solve the output minimum variance problem 
Proposition 1: An optimal solution to Problem (16) with $\omega \neq 0, \pi$ is given by

$$
\bar{u}(t)=\frac{\sqrt{2 \kappa \gamma} \sigma}{\sqrt{N}} \frac{\sqrt{2}}{\left|F\left(e^{i \omega}, \theta_{o}\right)\right|^{2}} \cos (\omega t),
$$

and thus

$$
u(t)=\frac{2 \sqrt{\kappa \gamma} \sigma}{\sqrt{N}} \cos (\omega t) .
$$

The solutions for $\omega=0, \pi$ are

$$
u(t)=\frac{\sqrt{2 \kappa \gamma} \sigma}{\sqrt{N}} \cos (\omega t), \quad \omega=0, \pi .
$$
satisfies

Proof: The covariance matrix of proposed input signal

$$
\bar{R}_{2 n}^{o p t}=\frac{\kappa \sigma^{2} \gamma}{N} \frac{2}{\left|F\left(e^{i \omega}, \theta_{o}\right)\right|^{4}} M
$$

and since $\bar{R}_{2 n} \geq \bar{R}_{2 n}^{\text {opt }}$ for all other feasible solutions we have

$$
\bar{b}^{T} \bar{R}_{2 n} \bar{b}_{o} \geq \bar{b}_{o}^{T} \bar{R}_{2 n}^{o p t} \bar{b}_{o}
$$

i.e. the cost function is minimized for $\bar{R}_{2 n}^{o p t}$. Notice that the solution does not depend on the phase of the sinusoidal signal. For $\omega=0, \pi$ we need to scale the amplitude with a factor of $\sqrt{2}$ to obtain the correct power.

Proposition 1 may seem obvious, but as shown in [24], it is for example in general not true if we instead use the constraint

$$
\mathrm{E}\left\{\left|G\left(e^{i \omega}, \hat{\theta}_{N}\right)-G\left(e^{i \omega}, \theta_{o}\right)\right|^{2}\right\} \leq \delta
$$

in the minimum variance optimization problem. The input minimum variance problem (15) is also more involved, since the dimension is higher and we have two matrix inequalities. The sinusoidal solution to Problem (16) satisfies the constraint, but as shown in the following example it is possible to find signals with a smaller optimal cost.

\section{A. Example of Input Minimum Variance Design}

Consider the first order OE frequency reponse

$$
G\left(e^{i \omega}\right)=\frac{b e^{-i \omega}}{1+f e^{-i \omega}}, \quad|f| \leq 1
$$

The minimum variance problem (15) then equals

$$
\begin{aligned}
\min _{\bar{r}_{k}, k=0,1,2} & {\left[1,2 f_{o}, f_{o}^{2}\right]\left[\begin{array}{lll}
\bar{r}_{0} & \bar{r}_{1} & \bar{r}_{2} \\
\bar{r}_{1} & \bar{r}_{0} & \bar{r}_{0} \\
\bar{r}_{2} & \bar{r}_{1} & \bar{r}_{0}
\end{array}\right]\left[1,2 f_{o}, f_{o}^{2}\right]^{T} } \\
\text { s.t. } \quad & {\left[\begin{array}{ll}
\bar{r}_{0} & \bar{r}_{1} \\
\bar{r}_{1} & \bar{r}_{0}
\end{array}\right] \geq C\left[\begin{array}{ccc}
1 & \cos (\omega) \\
\cos (\omega) & 1
\end{array}\right], } \\
& {\left[\begin{array}{lll}
\bar{r}_{0} & \bar{r}_{1} & \bar{r}_{2} \\
\bar{r}_{1} & \bar{r}_{0} & \bar{r}_{0} \\
\bar{r}_{2} & \bar{r}_{1} & \bar{r}_{0}
\end{array}\right] \geq 0, \quad C=\frac{\kappa \sigma^{2} \gamma}{N} \frac{2}{\left|F\left(e^{i \omega}, \theta_{o}\right)\right|^{4}} . }
\end{aligned}
$$

It is possible to analytically solve this problem. The solution is given in the appendix. The optimal solution depends on the inequalities

$$
\frac{2|f|(1-\cos (\omega))}{(1+f)^{2}} \geq 1, \quad \frac{2|f|(1+\cos (\omega))}{(1-f)^{2}} \geq 1 .
$$

If none of these constraints are fulfilled the optimal solution is the same as for the output variance case, i.e. the sinusoidal signal given by Proposition 1 . This is the case for small $|f|$, which makes sense since for $f=0$ the input variance and the output variance problem coincides. It is interesting to notice that a $|f|$ close to one may give a quite different solution. The following numerical example confirms this.

The true parameters are $b_{o}=1$ and $f_{o}=0.9$. Figure 1 shows the corresponding input minimum variance of the solution to Problem (15) and Problem (16) for $\omega \in[0, \pi]$. We have scaled the variances so that $r_{0}$ for the solution given by Proposition 1 is equal to 1 .

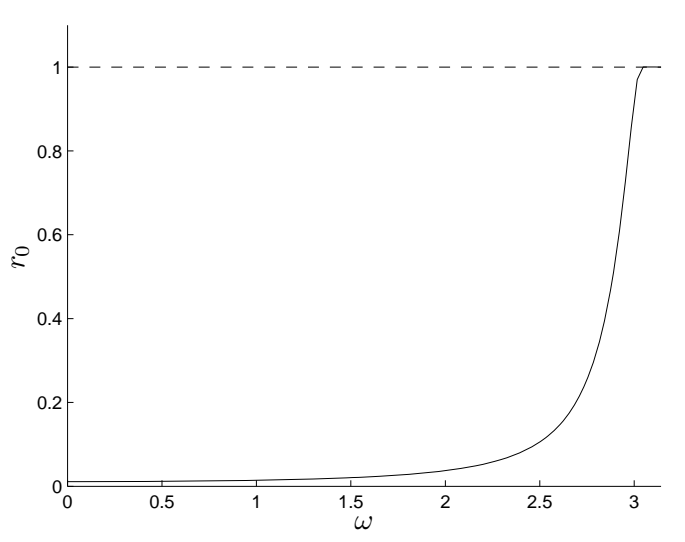

Fig. 1. First order example:Scaled input minimum variance of the solution to Problem (16) (dashed line) and to Problem (15) (full line) for $\omega \in[0, \pi]$.

\section{FEED Forward CONTROL EXAMPLES}

Consider the OE system with additive disturbance

$$
y(t)=G\left(q, \theta_{o}\right) u(t)+d(t),
$$

with input signal sequence $\{u(t)\}$, output signal sequence $\{y(t)\}$, and disturbance $\{d(t)\}$. The control objective is to reject the disturbance $d(t)$, i.e. to keep $y(t)$ close to zero. To simplify the calculations we will assume $d(t)$ to be a unit step disturbance,

$$
d(t)=\left\{\begin{array}{ll}
0, & t<0 \\
1, & t \geq 0
\end{array} .\right.
$$

The model based feed forward open loop controller to this disturbance rejection problem is $u(t)=-d(t) / G(1, \theta)$. We will study the effects of errors in the static gain $G(1, \theta)$ on the steady state control error using the application cost function

$$
V_{a p p}(\theta)=\left[1-\frac{G\left(1, \theta_{o}\right)}{G(1, \theta)}\right]^{2}
$$

Here

$$
\begin{aligned}
& V_{a p p}^{\prime \prime}\left(\theta_{o}\right)=\frac{2}{B^{2}\left(1, \theta_{o}\right) F^{2}\left(1, \theta_{o}\right)} \times \\
& \mathscr{S}\left(F_{o}, B_{o}\right)[1,1, \ldots, 1]^{T}[1,1, \ldots, 1] \mathscr{S}^{T}\left(F_{o}, B_{o}\right) .
\end{aligned}
$$

From Proposition 1 it follows that the constant signal

$$
\bar{u}(t)=\frac{\sqrt{2}}{B\left(\left(1, \theta_{o}\right) F\left(1, \theta_{o}\right)\right.} \frac{\sqrt{2 \kappa \gamma} \sigma}{\sqrt{N}}
$$

and hence

$$
u(t)=\frac{\sqrt{2}}{G(1, \theta)} \frac{\sqrt{2 \kappa \gamma} \sigma}{\sqrt{N}}
$$

solves the output minimum variance problem (13). 


\section{A Model Predictive Control Example}

The final examples concern Model Predictive Control (MPC). Here it is not possible to analytically find $V_{a p p}^{\prime \prime}(\theta)$, and we will instead use numerical differentiation. We will study two cases. In the first one we have a constant disturbance on the output signal and in the second one the disturbance is applied to the input.

\section{A. Constant Output Disturbance}

Consider a first order OE state space model with additive output disturbance $d(t)$ difference form

$$
\begin{aligned}
{\left[\begin{array}{l}
x_{1}(t+1) \\
x_{2}(t+1) \\
x_{3}(t+1)
\end{array}\right] } & =\left[\begin{array}{ccc}
1-f & f & -b \\
1 & 0 & 0 \\
0 & 0 & 0
\end{array}\right]\left[\begin{array}{l}
x_{1}(t) \\
x_{2}(t) \\
x_{3}(t)
\end{array}\right]+\left[\begin{array}{l}
b \\
0 \\
1
\end{array}\right] u(t) \\
+ & +\left[\begin{array}{l}
1 \\
0 \\
0
\end{array}\right] v(t) \\
y(t) & =\left[\begin{array}{lll}
1 & 0 & 0
\end{array}\right]\left[\begin{array}{l}
x_{1}(t) \\
x_{2}(t) \\
x_{3}(t)
\end{array}\right]
\end{aligned}
$$

where the state $x(t)=[y(t), y(t-1), u(t-1)]^{T}$ is fully measurable. Here $v(t)=d(t+1)+(f-1) d(t)-f d(t-1)$. The disturbance is $d(t)=1, t \geq 0$, and zero otherwise. This means that $v(t)=0, t \geq 1$. We then have $y(-1)=u(-1)=0$ and $y(0)=1$. The application cost-function is defined by

$$
V_{\text {app }}(\theta)=\frac{1}{M} \sum_{t=1}^{M}\left[y(t, \theta)-y\left(t, \theta_{o}\right)\right]^{2},
$$

where $y(t, \theta)$ is the output signal under the MPC feedback control based on the model $\theta$. The application set $\mathscr{E}_{a p p}$ is defined by (3), and the system identification set $\mathscr{E}_{S I}$ is defined by (4).

We use a standard MPC problem formulation

$$
\begin{array}{r}
\min _{u(k), k=t, \ldots t+h-1} \sum_{k=t+1}^{t+h}\left\{y^{2}(k)+\lambda[u(k-1)-u(k-2)]^{2}\right\} \\
+x^{T}(t+h) Q x(t+h)
\end{array}
$$

subject to the dynamics with given initial conditions $x(t)$ and a constraint on the input signal.

We will first study the system with true parameters $b_{o}=$ 0.1 and $f_{o}=-0.9$, i.e., the static gain of the system is 1 . The MPC prediction and control horizons are $h=10$ and $V_{a p p}$ is calculated with $M=10$. There are no constraints on the input signal in the MPC control problem. The weights $\lambda$ and $Q$ are both set to zero. The Hessian $V_{a p p}^{\prime \prime}\left(\theta_{o}\right)$ is found using numerical differentiation. The problem (12) is then solved with $\gamma=1000, \sigma^{2}=0.01, N=100$ and $\kappa$ from the $\chi^{2}(2)$ distribution with $\alpha=0.95$. We assume that the disturbance is not active during the identification experiment. We add, however, white measurement noise.

Figure 2 shows the ellipsoidal sets, where $\mathscr{E}_{S I}$ lies inside of $\mathscr{E}_{\text {app }}$. The larger semi-axis of $\mathscr{E}_{a p p}$ corresponds to eigenvector $[0.07-1.00]^{T}$ with eigenvalue 0.04 , and the smaller to eigenvector $\left[\begin{array}{ll}-1.00 & -0.07\end{array}\right]^{T}$ with eigenvalue 223 . We performed 1000 estimates of the model parameters, $94.6 \%$ of those are in $\mathscr{E}_{S I}$. We see that, with respect to the intended application, it is more important to estimate $b$ with high accuracy than $f$.

We now change the input weight $\lambda$ to 0.1 . The larger semiaxis of $\mathscr{E}_{a p p}$ corresponds to eigenvector $[0.07-1.00]^{T}$ with

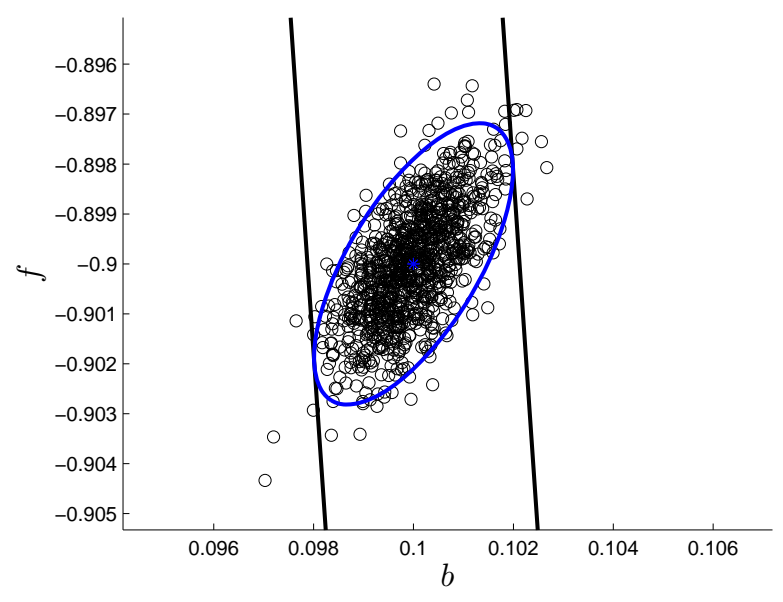

Fig. 2. MPC Example with $\lambda=0$ and output disturbance: $\mathscr{E}_{a p p}$ is the outer ellipse (almost vertical lines), $\mathscr{E}_{S I}$ is the inner ellipse and $\hat{\theta}_{N}$ are the small circles.

eigenvalue 0.05 , and the smaller to eigenvector $[-1.00-$ $0.07]^{T}$ with eigenvalue 41.4 . We can conclude that it is still more important to estimate $b$.

\section{B. Constant Input Disturbance}

We will now move the disturbance to the input of the system

$$
y(t)=\frac{b q^{-1}}{1+f q^{-1}}[u(t)+d(t)] .
$$

The incremental state space model (22) is modified to $v(t)=$ $b[d(t)-d(t-1)]$ with $d(t)=1, t \geq 0$ and zero otherwise. Here we will have $x(0)=0$. The application cost (23) and the MPC design formulation (24) remain the same and the settings are equivalent to those in the first example with output disturbance. The identification experiment is done without disturbance, but with measurement noise.

Figure 3 shows the obtained ellipsoidal sets. The larger semi-axis of $\mathscr{E}_{a p p}$ corresponds to eigenvector $[0.06-1.00]^{T}$ with eigenvalue 0.0005 , and the smaller to eigenvector $\left[\begin{array}{ll}-1.00 & -0.06\end{array}\right]^{T}$ with eigenvalue 0.89 . We performed 1000 estimates of the model parameters, $95 \%$ of those lie inside $\mathscr{E}_{S I}$. We can see that even though the disturbance has shifted from the output to the input, the most important direction remains the same. That is, it is still more crucial to estimate $b$ with high accuracy than $f$. Although, not as high accuracy as in the previous examples.

We can see that the important direction of the parameters has shifted compared to the output disturbance case. It is now crucial to estimate the positive weighted difference $0.81 f-0.58 b$ of the parameters with high accuracy instead of a positive weighted sum.

\section{CONCLUSION}

We have studied the system identification input design approach developed in [11] for OE models. By using the parametrization proposed in [22] we can relate the OE error case to input design for FIR models. The main difference is a more complicated cost function. The usefulness of the proposed approach lies in its simplicity and exactness. This is because many approaches to optimal input design rely 


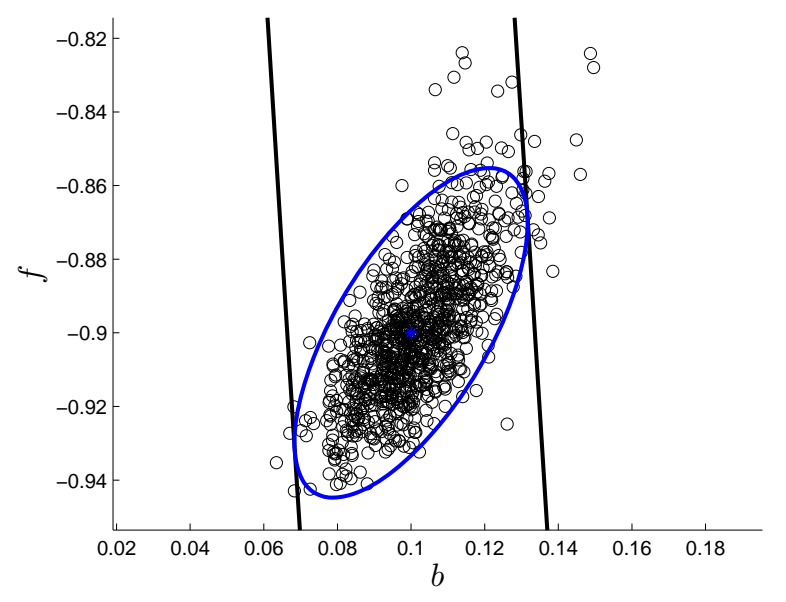

Fig. 3. MPC Example with $\lambda=0$ and input disturbance: $\mathscr{E}_{a p p}$ is the outer ellipse (almost vertical lines), $\mathscr{E}_{S I}$ is the inner ellipse and $\hat{\theta}_{N}$ are the small circles. .

either on the discretization of the spectrum, or on restricting its structure (say, to an MA spectrum). The so-called partial correlation approach [14], does not constrain the structure of the input spectrum, but it is a bit cumbersome to apply to general input design problems, since it cannot easily handle frequency domain constraints. The approach considered here, on the other hand, is reasonably straightforward to use and it has wide applicability, as we have shown in Sections III-V.

An interesting observation based on the parametrization introduced in Section II (based on [22]) is that for an important class of polynomial model structures (e.g. FIR, $\mathrm{OE}$ and $\mathrm{BJ}$, as seen here), the covariance matrix of the parameters, and thus their confidence ellipsoids, is constrained by a Toeplitz structure. This is an early observation in the system identification literature (since it motivated the original definition of "persistency of excitation" in terms of the positive definiteness of a Toeplitz matrix). However, it is important to remind the reader that this is the reason why it is impossible in many cases for the identification ellipsoid to match the application ellipsoid. Therefore, in order to understand the effects of the model structure in optimal input design (and identification in general), it is very important to analyze the geometry of Toeplitz ellipsoids, for which it is enough to start from the study of basic FIR structures, since the geometry of more complicated structures has similar degrees of freedom.

In addition, from the parametrization introduced in Section II we can clearly see the influence of the true parameter vector on the input design problem (for $\mathrm{OE}$ and $\mathrm{BJ}$ structures): the elements of $\theta_{o}$ appear in the Sylvester matrix $\mathscr{S}(F,-B)$ and the transformation $F^{2}(q) \bar{u}(t)=u(t)$. This shows that $\theta_{o}$ has a double effect: it changes the geometry of the confidence ellipsoid (i.e. the directions and lengths of its principal axes), and it affects the weighting of the spectral components of $u(t)$, in the sense that some frequencies will have more influence on the estimation accuracy than others. Understanding these effects can have great impact on areas such as robust experiment design, where the lack of knowledge about the true plant (before the experiment is performed) has to be explicitly taken into account.

\section{APPENDIX}

We will derive optimality conditions for problem (18) for

$$
C=\frac{\kappa \sigma^{2} \gamma}{N} \frac{2}{\mid F\left(\left.e^{i \omega}\right|^{4}\right.}=1
$$

The general solution is then obtained by multiplying the solution for $C=1$ with $C$. We will use the notation $d=$ $\cos (\omega) \in[-1,1]$. The objective function in (18) equals

$$
J\left(\bar{r}_{0}, \bar{r}_{1}, \bar{r}_{2}\right)=a \bar{r}_{0}+b \bar{r}_{1}+c \bar{r}_{2},
$$

with $a=1+4 f^{2}+f^{4}>0, b=4 f+4 f^{3}$ and $c=2 f^{2} \geq 0$, where $|f| \leq 1$. Notice that $a+b+c=(1+f)^{4}$ and $a-b+c=$ $(1-f)^{4}$. The first constraint in (18) is

$$
\left[\begin{array}{ll}
\bar{r}_{0}-1 & \bar{r}_{1}-d \\
\bar{r}_{1}-d & \bar{r}_{0}-1
\end{array}\right] \geq 0
$$

for which

$$
\begin{aligned}
& {\left[\begin{array}{cc}
1 & 1 \\
-1 & 1
\end{array}\right]^{T}\left[\begin{array}{cc}
\bar{r}_{0}-1 & \bar{r}_{1}-d \\
\bar{r}_{1}-d & \bar{r}_{0}-1
\end{array}\right]\left[\begin{array}{cc}
1 & 1 \\
-1 & 1
\end{array}\right]} \\
& =\left[\begin{array}{cc}
2\left(\bar{r}_{0}-\bar{r}_{1}-1+d\right) & 0 \\
0 & 2\left(\bar{r}_{0}+\bar{r}_{1}-1-d\right)
\end{array}\right] \geq 0
\end{aligned}
$$

Hence we obtain the condition

$$
\bar{r}_{0} \geq 1+\left|\bar{r}_{1}-d\right|
$$

Next, we will use the same transformation idea to obtain

$$
\begin{aligned}
& {\left[\begin{array}{ccc}
1 & 0 & 1 \\
0 & 1 & 0 \\
-1 & 0 & 1
\end{array}\right]^{T}\left[\begin{array}{ccc}
\bar{r}_{0} & \bar{r}_{1} & \bar{r}_{2} \\
\bar{r}_{1} & \bar{r}_{0} & \bar{r}_{1} \\
\bar{r}_{2} & \bar{r}_{1} & \bar{r}_{0}
\end{array}\right]\left[\begin{array}{ccc}
1 & 0 & 1 \\
0 & 1 & 0 \\
-1 & 0 & 1
\end{array}\right]} \\
& =\left[\begin{array}{ccc}
2\left(\bar{r}_{0}-\bar{r}_{2}\right) & 0 & 0 \\
0 & \bar{r}_{0} & 2 \bar{r}_{1} \\
0 & 2 \bar{r}_{1} & 2\left(\bar{r}_{0}+\bar{r}_{2}\right)
\end{array}\right] \geq 0
\end{aligned}
$$

This gives the two conditions

$$
\bar{r}_{0} \geq \bar{r}_{2}, \quad\left[\begin{array}{cc}
\bar{r}_{0} & 2 \bar{r}_{1} \\
2 \bar{r}_{1} & 2\left(\bar{r}_{0}+\bar{r}_{2}\right)
\end{array}\right] \geq 0
$$

The matrix inequality holds if

$$
\bar{r}_{0} \geq 0, \quad \bar{r}_{0}+\bar{r}_{2} \geq 0, \quad 2\left(\bar{r}_{0}^{2}+\bar{r}_{0} \bar{r}_{2}\right)-4 \bar{r}_{1}^{2} \geq 0
$$

The two conditions (29) can thus be summarized by

$$
\bar{r}_{0} \geq\left|\bar{r}_{2}\right|, \quad \bar{r}_{2} \geq \frac{2 \bar{r}_{1}^{2}}{\bar{r}_{0}}-\bar{r}_{0}
$$

We can now divide the derivation into three cases when the different conditions are active:

Case 1: Assume $\bar{r}_{1}>d$. Condition (27) is then active for $\bar{r}_{0}=1+\bar{r}_{1}-d \Rightarrow \bar{r}_{1}=\bar{r}_{0}-1+d$. Condition (30) then implies

$$
\bar{r}_{2} \geq \frac{2 \bar{r}_{1}^{2}}{\bar{r}_{0}}-\bar{r}_{0}=\bar{r}_{0}+\frac{2(1-d)^{2}}{\bar{r}_{0}}-4(1-d)
$$

and the cost-function (25) with these constraints active becomes

$$
J\left(\bar{r}_{0}\right)=a \bar{r}_{0}+b\left(\bar{r}_{0}-1+d\right)+c\left(\bar{r}_{0}+\frac{2(1-d)^{2}}{\bar{r}_{0}}-4(1-d)\right)
$$

This is a convex function in $\bar{r}_{0}$ and minimized for

$$
\bar{r}_{0}^{2}=\frac{2 c(1-d)^{2}}{a+b+c}=\frac{4 f^{2}(1-\cos (\omega))^{2}}{(1+f)^{4}}
$$


This solution is only valid if $\bar{r}_{1}>d$, which is equivalent to $\bar{r}_{0}>1$. This means

$$
\begin{aligned}
& \frac{2|f|(1-\cos (\omega))}{(1+f)^{2}}>1 \Leftrightarrow \Leftrightarrow \\
&|f-(-2+\cos (\omega))|<\sqrt{(2-\cos (\omega))^{2}-1} .
\end{aligned}
$$

Case 2: Assume $\bar{r}_{1}<d$. Condition (27) is then active for $\bar{r}_{0}=1-\bar{r}_{1}+d \Rightarrow \bar{r}_{1}=-\bar{r}_{0}+1+d$. Condition (30) then implies

$$
\bar{r}_{2} \geq \frac{2 \bar{r}_{1}^{2}}{\bar{r}_{0}}-\bar{r}_{0}=\bar{r}_{0}+\frac{2(1+d)^{2}}{\bar{r}_{0}}-4(1+d)
$$

and the cost-function (25) with these constraints active becomes

$$
J\left(\bar{r}_{0}\right)=a \bar{r}_{0}+b\left(-\bar{r}_{0}+1+d\right)+c\left(\bar{r}_{0}+\frac{2(1+d)^{2}}{\bar{r}_{0}}-4(1+d)\right)
$$

This is a convex function in $\bar{r}_{0}$ and minimized for

$$
\bar{r}_{0}^{2}=\frac{2 c(1+d)^{2}}{a-b+c}=\frac{4 f^{2}(1+\cos (\omega))^{2}}{(1-f)^{4}}
$$

This solution is only valid if $\bar{r}_{1}<d$, which is equivalent to $\bar{r}_{0}>1$, i.e.

$$
\begin{array}{r}
\frac{2|f|(1+\cos (\omega))}{(1-f)^{2}}>1 \Leftrightarrow \\
\mid f-\left(2+\cos (\omega) \mid<\sqrt{(2+\cos (\omega))^{2}-1} .\right.
\end{array}
$$

Case 3: Finally, we now have to check the case $\bar{r}_{1}=d$ and $\bar{r}_{0}=1$. Since $c \geq 0$ in the cost function (25), $\bar{r}_{2}$ should be chosen as small as possible, but (30) implies $\bar{r}_{2} \geq \frac{2 \bar{r}_{1}^{2}}{\bar{r}_{0}}-\bar{r}_{0}=$ $2 d^{2}-1=\cos (2 \omega)$. The condition $\bar{r}_{0} \geq\left|\bar{r}_{2}\right|$ will only be active for $\omega=0, \pi$.

Summary: Inequality (31) defines an interval for $f$ centered at $f=-2+\cos (\omega)$ (for fixed $\omega$ ), which is contained in $-1<f<0$. The interval contain $f=-1$ but not $f=$ 0 becomes $(-2+\cos (\omega))^{2} \leq(2-\cos (\omega))^{2}-1$, which is a contradiction. Inequality (32) defines an interval for $f$ centered at $f=2-\cos (\omega)$ (for fixed $\omega$ ), which is contained in $0<f<1$. The interval contains $f=1$, but not $f=0$.

Depending on $f$, and $\omega$ we have the optimal solutions:

If (31) holds (negative $f$ ), the optimal solution is

$$
\begin{aligned}
& \bar{r}_{0}=\frac{2|f|(1-\cos (\omega))}{(1+f)^{2}}, \bar{r}_{1}=\bar{r}_{0}-1+\cos (\omega), \\
& \bar{r}_{2}=\bar{r}_{0}+\frac{2(1-\cos (\omega))^{2}}{\bar{r}_{0}}-4(1-\cos (\omega)) .
\end{aligned}
$$

If (32) holds (positive $f$ ), the optimal solution is

$$
\begin{aligned}
& \bar{r}_{0}=\frac{2|f|(1+\cos (\omega))}{(1-f)^{2}}, \bar{r}_{1}=-\bar{r}_{0}+1+\cos (\omega), \\
& \bar{r}_{2}=\bar{r}_{0}+\frac{2(1+\cos (\omega))^{2}}{\bar{r}_{0}}-4(1+\cos (\omega))
\end{aligned}
$$

If neither of the conditions hold (small $f$ ) the optimal solution is

$$
\bar{r}_{0}=1, \bar{r}_{1}=\cos (\omega), \bar{r}_{2}=\cos (2 \omega),
$$

\section{REFERENCES}

[1] M. Barenthin, X. Bombois, H. Hjalmarsson, and G. Scorletti. Identification for control of multivariable systems: Controller validation and experiment design via LMIs. Automatica, 44(12):3070-3078, 2008.

[2] M. Barenthin, H. Jansson, H. Hjalmarsson, J. Mårtensson, and B. Wahlberg. A control perspective on optimal input design in system identification. In Forever Ljung in System Identification, chapter 10. Studentlitteratur, September 2006.

[3] G. Belforte and P. Gay. Optimal experiment design for regression polynomial models identification. International Journal of Control, 75(15):1178-1189, 2002.

[4] X. Bombois and H. Hjalmarsson. Optimal input design for robust $\mathrm{H}_{2}$ deconvolution filtering. In 15th IFAC Symposium on System Identification, Saint-Malo, France, July 2009. To appear.

[5] X. Bombois, G. Scorletti, M. Gevers, P. M. J. Van den Hof, and R. Hildebrand. Least costly identification experiment for control. Automatica, 42(10):1651-1662, 2006.

[6] B.L. Cooley, J.H. Lee, and S.P. Boyd. Control-relevant experiment design: a plant-friendly, lmi-based approach. In American Control Conference, 1998. Proceedings of the 1998, volume 2, pages 12401244, June 1998.

[7] G. Franceschini and S. Macchietto. Model-based design of experiments for parameter precision: State of the art. Chemical Engineering Science, 63(19):4846 - 4872, 2008.

[8] L. Vandenberghe S. Boyd G. Javorzky, I. Kollar and S.-P. Wu. Optimal excitation signal design for frequency domain system identification using semidefinite programming. In Proceedings of the 8th IMEKO TC4 Symposium on Recent Advances in Electrical Measurements, Budapest, Hungary, pages 192-197, 1996.

[9] R. Hildebrand and M. Gevers. Identification for control: Optimal input design with respect to a worst-case $v$-gap cost function. SIAM $J$. Control Optim, 41(5):1586-1608, 2003.

[10] R. Hildebrand and G. Solari. Identification for control: Optimal input intended to identify a minimum variance controller. Automatica, 43(5):758-767, 2007.

[11] H. Hjalmarsson. System identification of complex and structured systems. European Journal of Control, pages 275-2310, 2009.

[12] H. Hjalmarsson and H. Jansson. Closed loop experiment design for linear time invariant dynamical systems via LMIs. Automatica, 44(3):623-636, 2008

[13] H. Hjalmarsson and J. Mårtensson. Optimal input design for identification of non-linear systems: Learning from the linear case. In American Control Conference, New York City, USA, July 11-13 2007.

[14] H. Jansson and H. Hjalmarsson. Input design via LMIs admitting frequency-wise model specifications in confidence regions. IEEE Transactions on Automatic Control, 50(10):1534-1549, 2005.

[15] C. Jauberthie, L. Denis-Vidal, P. Coton, and G. Joly-Blanchard. An optimal input design procedure. Automatica, 42(5):881 - 884, 2006.

[16] K. Lindqvist and H. Hjalmarsson. Optimal input design using linear matrix inequalities. In IFAC Symposium on System Identification, 2000.

[17] L. Ljung. System Identification - Theory For the User, 2nd ed. Prentice Hall, Upper Saddle River, New Jersey, 1999.

[18] T.S. Ng, G. Goodwin, and T. Söderström. Optimal experiment design for linear systems with input-output constraints. Automatica, (6):571577, 1977.

[19] L. Pronzato. Optimal experimental design and some related control problems. Automatica, 44(2):303-325, February 2008.

[20] C. R. Rojas, J. C. Agüero, J. S. Welsh, and G. C. Goodwin. On the equivalence of least costly and traditional experiment design for control. Automatica, 44(11):2706-2715, 2008

[21] C. R Rojas, J. S. Welsh, G. C. Goodwin, and A. Feuer. Robust optimal experiment design for system identification. Automatica, 43(6):9931008, June 2007.

[22] P. Stoica and T. Söderström. A Useful Parameterization for Optimal Experiment Design. IEEE Transactions on Automatic Control, AC27(4):986-989, August 1982.

[23] B. Wahlberg, H. Hjalmarsson, and M. Annergren. On optimal input design in system identification for control. In Proceedings IEEE Conference on Decision and Control, pages 5548-5552, December 2010.

[24] B. Wahlberg, H. Hjalmarsson, and P. Stoica. On optimal input design for frequency response estimation. In Proceedings 49th IEEE Conference on Decision and Control, pages 302-307, Atlanta, GA, USA, December 2010. 